\title{
Phase II study of ACNU in non-small-cell lung cancer: EORTC study 08872
}

\author{
A. S. Th. Planting ${ }^{1}$, A. Ardizzoni ${ }^{2}$, J. Estapé3, G. Giaccone ${ }^{4}$, G. Scagliotti ${ }^{5}$, T. A. W. Splinter ${ }^{6}$, A. Kirkpatrick ${ }^{7}$, \\ O. Dalesio ${ }^{8}$, and J. G. McVie ${ }^{9}$ \\ ${ }^{1}$ Dr Daniel den Hoed Cancer Center, Rotterdam, The Netherlands, ${ }^{2}$ Instituto Nazionale per la Ricerca Sul Cancro, Genova, Italy, \\ ${ }^{3}$ Hospital Clinic I Provincial de Barcelona, Barcelona, Spain, ${ }^{4}$ Hospital San Giovanni, Torino, Italy, ${ }^{5}$ S. Luigi Gonzaga Hospital, University of \\ Torino, Italy, ${ }^{6}$ University Hospital Dijkzigt, Rotterdam, The Netherlands, ${ }^{7}$ Data Manager EORTC, Brussels, Belgium. \\ ${ }^{8}$ Statistician EORTC, Brussels, Belgium, ${ }^{9}$ Cancer Research Campaign, London, United Kingdom
}

Received 2 June 1990/Accepted 14 January 1991

Summary. A total of 62 patients with metastatic or locally advanced non-small-cell lung cancer were entered in a phase II study of ACNU. Initially, the drug was given i.v. at a dose of $100 \mathrm{mg} / \mathrm{m}^{2}$ every 6 weeks, but due to observed haematological side effects in chemotherapy-pretreated patients, the dose was lowered in this group to $75 \mathrm{mg} / \mathrm{m}^{2}$. We observed one complete response in a subject exhibiting multiple lung metastases and a partial response in two patients, one showing brain metastases and one who experienced local disease recurrence. The toxicity of ACNU mainly consisted of bone marrow suppression especially thrombocytopenia, with one toxic death occurring due to intracerebral haemorrhage. We concluded that at this dose and on this schedule, ACNU has limited activity in nonsmall-cell lung cancer.

\section{Introduction}

ACNU [1-(4-amino-2-methyl-5-pyrimidinyl)-methyl-(2chloroethyl)-3-nitrosourea hydrochloride] is a water- and lipid-soluble nitrosourea derivative developed by Arakawa et al. in 1974 [1]. As an alkylating agent, it shows activity against transplanted human xenografts of $\mathrm{GxF} 1$ gastric cancer, MX1 breast cancer and $\mathrm{CO} 4$ colon cancer in nude mice [5]. Pharmacology studies in man reveal a plasma half-life of $35 \mathrm{~min}$ following intravenous administration. The drug is excreted via the bile and urine.

In a phase I study performed in Japan [2], dose-limiting toxicities consisted of granulocytopenia and thrombocytopenia; other toxicities observed included nausea, vomiting, and anorexia. Based on the results of this phase I study, a dose of $100 \mathrm{mg} / \mathrm{m}^{2}$ to be given at intervals of $6-8$ weeks was recommended.

Offprint requests to: A. S. Th. Planting, Department of Medical Oncology, Dr Daniel den. Hoed Cancer Center, Groene Hilledijk 301, 3075 EA Rotterdam, The Netherlands
The experience with ACNU in non-small-cell lung cancer is very limited. In a phase II study performed by Saijo and Niitani [3], a response was observed in 5 of 28 patients (17.9\%) with squamous-cell carcinoma of the lung. To confirm these results and to augment the current experience with ACNU in non-small-cell lung cancer, the EORTC Lung Cancer Cooperative Group decided to perform a phase II study of ACNU in non-small-cell lung cancer.

\section{Patients and methods}

ACNU was given as a slow i. v. infusion at a dose of $100 \mathrm{mg} / \mathrm{m}^{2}$ every 6 weeks. Due to haematological side effects observed in chemotherapypretreated patients, the dose was lowered during the study to $75 \mathrm{mg} / \mathrm{m}^{2}$ every 6 weeks in this group. In cases of bone marrow suppression, retreatment was delayed until haematological recovery; a dose reduction of $25 \%$ was carried out in patients showing a WBC nadir of $1-2 \times 10^{9} /$ and/or a platelet nadir of $50-75 \times 10^{9} / \mathrm{l}$. In subjects exhibiting a WBC nadir of $<1 \times 10^{9} / 1$ and/or a platelet nadir of $<50 \times 10^{9} /$, the dose was reduced to $50 \%$ of the previous level.

Patients in this study were required to have histologically proven non-small-cell lung cancer, locally advanced or metastastic, with measurable or evaluable lesions, documented progression within 2 months prior to entry into the study, an ECOG performance status of $\geqslant 2$, an age of $<70$ years, a WBC of $>4 \times 10 \%$, a platelet count of $>100 \times 10^{9} / 1$, a creatinine clearance of $>40 \mathrm{ml} / \mathrm{min}$ and a serum bilirubin level of $<2.5 \mathrm{mg} / \mathrm{dl}$. All patients underwent a chest X-ray and, if indicated, a chest and/or abdominal computer-assisted tomographic (CT) scan before the start of treatment. During treatment, estimations of haemoglobin, WBC and platelets were done weekly and liver and renal functions were evaluated every 6 weeks. Response to treatment was assessed every 6 weeks according to WHO criteria [7]; responses were reviewed extramurally.

\section{Results}

In this phase II study, pretreated and non-pretreated patients were analyzed separately. A total of 62 patients were entered; 34 subjects had not been pretreated and 28 had previously received various cisplatin-based chemotherapy regimens. 
Table 1. Patients' characteristics and treatment results

\begin{tabular}{ll}
\hline Patients entered & 62 \\
No previous chemotherapy & 34 \\
Previous chemotherapy & 28 \\
Men: Women & $53: 9$ \\
Median age (range) & $59(41-73)$ years \\
Median WHO performance status (range) & $1(0-2)$ \\
Histology: & 29 \\
Squamous cell carcinoma & 25 \\
Adenocarcinoma & 6 \\
Large-cell undifferentiated & $1(1-9)$ \\
Median number of courses given (range) & 1 \\
Complete response & 2 \\
Partial response & $8^{\mathrm{a}}$ \\
Stable disease & 39 \\
Progressive disease & \\
Early death: & 3 \\
Malignant disease & 3 \\
Other causes & 6 \\
Nonevaluable & \\
\hline
\end{tabular}

a 1 toxic death

The patients' characteristics are summarized in Table 1. Overall, 50 subjects were fully evaluable for response and toxicity; 6 patients died before the first evaluation, scheduled at 6 weeks after the first ACNU dose: 3 due to rapidly progressive disease and 3 due to causes not related to tumor or treatment. Another 6 patients were not fully evaluable: therapy was stopped in 1 case because of severe thrombocytopenia at 4 weeks after the first dose (this patient was included in the toxicity analysis) and in 1 subject because of intercurrent gastro-intestinal disease; 1 patient received an inadequate dose and 3 refused further treatment after the first course.

Initially, 11 pretreated patients were started on an ACNU dose of $100 \mathrm{mg} / \mathrm{m}^{2}$; as 2 cases of grade 3 and 4 cases of grade 4 thrombocytopenia were observed, it was decided to lower the ACNU dose to $75 \mathrm{mg} / \mathrm{m}^{2}$ in this patient group. We observed a complete response in 1 patient with multiple lung metastases from an adenocarcinoma, which lasted for 9 months, and 2 partial responses: 1 in a local recurrence of an adenocarcinoma (for a duration of 9 months) and 1 in brain metastases from a squamous-cell carcinoma that lasted for 2 months. Two patients had not previously been treated; the response in the local recurrence occurred in a previously heavily pretreated subject who was treated at a dose of $75 \mathrm{mg} / \mathrm{m}^{2}$. The overall response rate was $5 \%(95 \%$ confidence limits, $1.7 \%-22.4 \%$ ).

The observed toxicity consisted mainly of bone marrow depression, including granulocytopenia of grades 3 (four cases) and 4 (one patient) and thrombocytopenia of grades 3 (seven subjects) and 4 (eight cases). Seven patients required platelet transfusions. One toxic death occurred due to an intracerebral haemorrhage during grade 4 thrombocytopenia. After lowering the ACNU dose in pretreated subjects we observed only 1 case of grade 3 and 1 of grade 4 thrombocytopenia in 14 patients. In the non-pretreated patients, four cases of grade 3 and three of grade 4 thrombocytopenia were observed, in one patient during the first course and in the others during the second and third courses. WBC nadirs occurred at week 4 and platelet nadirs, at week 5 , causing treatment delays in five courses. Other toxicities observed were nausea and vomiting (grade 3 in 4 patients) and alopecia (grade 1 in 12 subjects).

\section{Discussion}

Prior to the cisplatin era, nitrosourea derivatives were frequently incorporated in chemotherapy regimens for non-small-cell lung cancer. CCNU and methyl-CCNU show some activity as single agents [6] but have the disadvantage of being lipid-soluble and must therefore be given by mouth. ACNU is lipid- and water-soluble and can be safely given intravenously. Apart from bone marrow toxicity, ACNU produces only minor gastrointestinal side effects; it lacks nephrotoxicity and pulmonary toxicity, in contrast to methyl-CCNU and BCNU. Because of these characteristics and the response rate of $17.8 \%$ previously observed in squamous-cell lung cancer [3], our group considered it worthwhile to study ACNU in all histological subtypes of lung cancer. On the schedule used in the present study, we could not verify the results obtained by Saijo and Niitani [3]; we observed 1 complete and 2 partial responses in 50 evaluable patients. Using a slightly lower dose, Sasaki et al. [4] observed no responders among 21 patients. The better results of Saijo and Niitani [3] cannot be explained by selection of histology. In our study, only 1 of 29 patients with squamous-cell carcinoma responded. The ACNU toxicity observed in this study consisted mainly of thrombocytopenia and tended to increase with continuation of treatment. We conclude that the activity of ACNU in non-small-cell lung cancer is negligible at this dose and on this schedule.

Acknowledgements. ACNU was kindly supplied by ASTA-Pharma Aktiengesellschaft (FRG).

\section{References}

1. Arakawa M, Shimizu F, Okada N (1974) Effect of 1-(4-amino-2methylpyrimidinyl)-methyl-3-(2-chloroethyl)-3-nitrosurea hydrochloride on leukemia L-1210. Gann 65: 191

2. Cooperative Study Group (1976) Phase I study of ACNU. Jpn J Clin Oncol 6: 55-62

3. Saijo N, Niitani H (1980) Experimental and clinical effect of ACNU in Japan, with emphasis on small-cell carcinoma of the lung. Cancer Chemother Pharmacol 4: 165-171

4. Sasaki Y, Saijo N, Shimuzu E (1985) Phase II study of ACNU for non-small-cell lung cancer. Eur J Cancer Clin Oncol 21: 1557-1559

5. Shimizu F, Arakawa M (1978) Antitumor activity of ACNU in a variety of experimental tumors. Gann 69: 545 - 548

6. Sorensen JB, Clerici M, Hansen HH (1988) Review. Single-agent chemotherapy for advanced adenocarcinoma of the lung. Cancer Chemother Pharmacol 21: 89-102

7. WHO (1979) Handbook for reporting results of cancer treatment. Offset Publication 45. WHO, Geneva 